International Journal of Bifurcation and Chaos, Vol. 26, No. 10 (2016) 1630026 (18 pages)

(c) The Author(s)

DOI: $10.1142 / \mathrm{S} 0218127416300263$

\title{
Switching Dynamics Between Two Movement Patterns Varies According to Time Interval
}

\author{
Takehito Hirakawa \\ Department of Psychology and Human Relations, \\ Nanzan University, Nagoya 466-8673, Japan \\ takehito@nanzan-u.ac.jp \\ Hiroo Suzuki \\ Faculty of Human Sciences, Takachiho University, \\ Suginami-ku 168-8508, Japan \\ hiroo@takachiho.ac.jp \\ Motoki Okumura \\ Faculty of Education, Tokyo Gakugei University, \\ Koganei 184-8501, Japan \\ okumura@u-gakugei.ac.jp \\ Kazutoshi Gohara \\ Division of Applied Physics, Hokkaido University, \\ Sapporo 060-8628, Japan \\ gohara@eng.hokudai.ac.jp \\ Yuji Yamamoto \\ Research Center of Health, Physical Fitness and Sports, \\ Nagoya University, Nagoya 464-8601, Japan \\ yamamoto@htc.nagoya-u.ac.jp
}

Received November 20, 2015; Revised May 23, 2016

\begin{abstract}
This study investigated the regularity that characterizes the behavior of dissipative dynamical systems excited by external temporal inputs for pointing movements. Right-handed healthy male participants were asked to continuously point their right index finger at two light-emitting diodes (LEDs) located in the oblique left and right directions in front of them. These movements were performed under two conditions: one in which the direction was repeated and one in which the directions were switched on a stochastic basis. These conditions consisted of 12 tempos (30, 36, $42,48,51,54,57,60,63,66,69$, and 72 beats per minute). Data from the conditions under which the input pattern was repeated revealed two different trajectories in hyper-cylindrical state space $\mathcal{M}$, whereas the conditions under which the inputs were switched induced transitions between the two trajectories, which were considered to be excited attractors. The transitions between the two excited attractors were characterized by a self-similar structure. Moreover, the correlation dimensions increased as the tempos increased. These results suggest a relationship of $D \propto 1 / T$ ( $T$ is the switching-time length; i.e. the condition) between temporal input and pointing behavior and that continuous pointing movements are regular rather than random noise.
\end{abstract}

Keywords: Switching dynamics; self-similarity; motor control; complex system.

This is an Open Access article published by World Scientific Publishing Company. It is distributed under the terms of the Creative Commons Attribution 4.0 (CC-BY) License. Further distribution of this work is permitted, provided the original work is properly cited. 


\section{Introduction}

\subsection{Dynamical system perspective on human motor control}

The purpose of this study was to examine the control of rhythmical movement by humans. Previous research analyzing such movement was based on the frameworks of motor program theory [Schmidt et al., 1979; Schmidt, 1991] and dynamical system theory [Haken et al., 1985; Kelso, 1984]. In the former, consecutive behavior was regarded as the result of repeated execution by a low-flexible motor program [Yamamoto \& Gohara, 2000]. In addition, the variability that occurred in consecutive behaviors was considered to consist of a stochastic Gaussian distribution or random variation, such as a uniform distribution and white noise. In the latter, the dynamical system approach, the variability that occurred in continuous human movement was assumed to be not random variation but partly structured. This structure of variability corresponds to the fractal-like structure or self-similarity structure in a dynamical (i.e. complex) system. Furthermore, variability is inherent within all biological systems and reflects variation in both space and time. As a central feature of normal movement, the optimal variability is consistent with a nonlinear approach [Harbourne \& Stergiou, 2009], that is, the dynamical system approach.

About 50 years have passed since geometric structures or arrangements, such as fractals or selfsimilarity, were discovered in various natural phenomena. Early well-known research included that of Mandelbrot [1967], who showed the fractal-like structure inherent in the British coastline. This analysis focused on spatial properties, and the fractal was identified as self-similar geometric objects [Werner, 2010]. Since then, fractal theory has been used to understand not only natural phenomena but also, from a biological viewpoint, the living body. For example, detrended fluctuation analysis has been used to perform detailed analyses in the temporal domain [Peng et al., 1994, 1995], and power spectrum density analysis has been used in the frequency domain [Ding et al., 2002; Torre et al., 2007]. Furthermore, in recent years, these analyses have been extended to not only human motor control research, such as in sports [Nakayama et al., 2010] and occupational and clinical biomechanics [Hausdorff, 2007; Kirchner et al., 2014], but also to timeseries analysis in football, as it involves coordination among many players and competition with the opposing team. Temporal fluctuations in both the team turf boundary and the ball location have been found to follow the rules of fractional Brownian motion [Kijima et al., 2014]. Consequently, selfsimilar structures have been identified in time-series data regarding various phenomena.

Until now, the complex systems that are typical of fractal-like or self-similar structures (e.g. physics, biology, and economics) have been considered to be composed of many subsystems. These subsystems interact with one another and with the external environment [Gohara \& Okuyama, 1999b]. This implies that we should not assume that only one system is involved in human motor control and that we should consider the relationships between subsystems and the environment. Such phenomena have also been observed in various physical manifestations other than human movement, such as optical systems [Arecchi et al., 1986; Tanii et al., 1991, 1999], turbulence [Constantin et al., 1991; Maas et al., 1997; Schmiegel \& Eckhardt, 1997] and electronic circuits [Matias et al., 1997; Mestl et al., 1997]. This subsystem in human motor control corresponds to a movement pattern, and the behavioral output of this pattern by the subsystem is from an attractor. This study examined the interrelationship among several attractors that contribute to the basis of the subsystem's behavior. Consequently, we hypothesized that there are common underlying dynamic characteristics and patterns across different systems, that are captured and described using this framework that is reliable in a dissipative dynamical system stochastically excited by temporal inputs [Gohara \& Okuyama, 1999b], and one that has been examined experimentally [Suzuki \& Yamamoto, 2015; Yamamoto \& Gohara, 2000]. We conducted this study within the framework of this theory.

Focusing on human motor control, Yamamoto and Gohara [2000] verified that the dynamical changes in behavior that were affected by movement patterns switched abruptly in a sequential fashion. This phenomenon is called switching dynamics. They examined the switching dynamics between two attractors that formed the movement patterns of the forehand and backhand strokes in tennis. Specifically, they analyzed time-series data from the two stroke motions in tennis and studied the geometric structure in the state space. As a result, they confirmed that the structure is the Cantor set. 


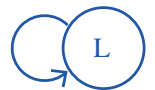

(a)

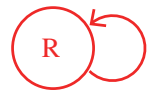

(b)

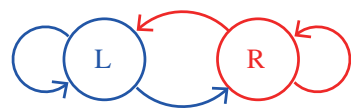

(c)
Fig. 1. Schematic diagrams of the interplay between systems. (a) A state in which a pointing-movement pattern toward the left $(\mathrm{L})$ side is continuously excited with temporal input (an autonomous system); (b) the right ( $\mathrm{R}$ ) side that is excited in the same way; (c) a mixed state in which only the $\mathrm{L}$ or $\mathrm{R}$ side is excited, but the $\mathrm{L}$ and $\mathrm{R}$ sides are mutually excited and affected (nonautonomous system).

Figures 1(a)-1(c) are schematic diagrams of these phenomena. For example, Figs. 1(a) and 1(b) imply continuous pointing movement excited by one attractor; that is, only one direction, either right or left, is repeated. Figure 1(c) implies that the dynamics between two attractors switch, and continuous movement is repeatedly switched to either the left or right side. If we try to understand continuous human movement according to motor program theory, we evaluate the output of human behavior as composed of continuous random trajectories. We examined human behavior with two external stimuli presented on the left and right sides; that is, we prepared a sequence of random stimuli [e.g. rightleft-left-right-left, etc.(RLLRL...)]. However, this sequence was classified as only eight external input patterns (RRR, LRR, RLR, LLR, LRL, RRL, RLL, and LLL) and had third-order sequences. If outputs from human movement patterns were random, this third-order sequence effect would not remain and the average trajectory of outputs would converge into one trajectory. If eight sequence effects remained and human movement were characterized by hysteresis, the average trajectory of outputs would be eight trajectories. As mentioned above, it has been confirmed that these eight trajectories exist in activities involving complex human motor control, such as sports [Yamamoto \& Gohara, 2000], and that the structure is self-similar, as if it were the Cantor set.

In a recent study, Suzuki and Yamamoto [2015] used a correlation dimension, which is one of the fractal dimensions used to quantify fractal-like or self-similar structures, to analyze the switching dynamics between two attractors that were inherent motion patterns in table tennis, examining whether the dynamics were affected by different temporal intervals (i.e. the switching-time length $T$ ). They showed that the dynamics had only a fractal-like structure, but the correlation dimension varied with skill level and $T$. In this study, we applied the theoretical model developed by Gohara and Okuyama [1999a, 1999b] to human motor control and elaborated on their findings [Suzuki \& Yamamoto, 2015; Yamamoto \& Gohara, 2000]. That is, we attempted to evaluate the switching dynamics between two attractors involved in human motor control by calculating the correlation dimension and demonstrating the regular relationship between switching-time length $T$ and the correlation dimension. We focused on a pointing task where participants pointed their index finger towards the left and right diagonal directions (left and right sides) in front of them. According to Yamamoto and Gohara [2000], if we move our finger continuously (i.e. a pointing movement) toward the left and right sides, we can observe that this pointing movement is composed of two movement patterns. That is, these movement patterns are composed of two attractors when we continuously and rapidly move our finger in two directions. Our main focus was on how the behavior switched stochastically and rapidly with switching-time length $T$, how it affected the correlation dimension, and how it changed the relationship between them. The purpose of this experiment was to examine how serial effects affect motor output when the input was switched stochastically and rapidly and to demonstrate the regularity of the relationship with regard to human motor control by analyzing the relationship between switching-time length $T$ and correlation dimensions.

\subsection{Mathematical background}

Previous analyses of the interrelationships among attractors in a complex system, especially those using a dynamical system approach, have been carried out using two different systems, autonomous and nonautonomous. The former is called a closed system; the latter is called an open system. Defining the systems by an ordinary differential equation, an autonomous dynamical system is expressed as $\dot{x}=f(x)$. A nonautonomous system, depending on the periodic temporal input $I(t)$, is generally defined by the following equation:

$$
\dot{x}=f[x, I(t)], \quad x, I \in R^{N}
$$

$x$ and $f$ are the state and the vector fields, respectively. Equation (1) implies that the temporal development of the state $x$ is influenced by the input $I(t)$ from other systems. An attractor excited by 
the external input $I(t)$ is called the excited attractor. In this theory, we should consider how the input $I(t)$ changes the vector field over time.

In a dissipative dynamical system with periodic input $I(t)=I(t+T)$, the introduction of the angular variable $\theta \equiv \frac{2 \pi}{T} t \bmod 2 \pi$ and a new state variable $y \equiv(x, \theta)$ can transform the nonautonomous system [Eq. (1)] into the following autonomous system [Gohara \& Okuyama, 1999b]. This system is called a continuous dynamical system

$$
\dot{y}=f_{I}(y), \quad y \in R^{N} \times S^{1}: \mathcal{M} .
$$

The hyper-cylindrical state space (i.e. state space) $\mathcal{M}$ is incorporated into the continuous dynamical system, and a solution $\phi$ starting from an initial state $x_{0}$ at $t=0(\theta=0)$ converges with an attractor at $t \rightarrow \infty$.

In the state space $\mathcal{M}$, we can define the Poincaré section $\sum=\left\{(x, \theta) \in R^{N} \times S^{1} \mid \theta=2 \pi\right\}$, where a trajectory starting from an initial state at $\theta=0$ returns to the same point at $\theta=2 \pi$. Introducing Poincaré section $\sum$ into the state space $\mathcal{M}$, we can define the following discrete dynamical system. In section $\sum$, a state $x_{\tau}$ is transformed into another state $x_{\tau+1}$ after an interval $T$ by the map $g_{I}$. Therefore, we can study two kinds of dynamics with a continuous dynamical system [Eq. (2)] and a discrete dynamical system [Eq. (3)]

$$
x_{\tau+1}=g_{I}\left(x_{\tau}\right), \quad x_{\tau} \in R^{N}: \sum .
$$

Moreover, any subsystems that interact with another subsystem because of the latter are assumed to be the external environment in the nonautonomous dynamical system [Gohara \& Okuyama, 1999a, 1999b; Nishikawa \& Gohara, 2002; Sato \& Gohara, 2001; Wada \& Gohara, 2001a, 2001b]. Consider the dynamics when multiple input patterns are stochastically and sequentially fed into the system. Let us consider the input as a set $\left\{I_{l}\right\}_{l=1}^{L}$ of time functions $I_{l}$ sampled on the parameterized space. The subscripts and individual sets are sometimes abbreviated and expressed as $\{\cdot\}$ for simplicity. When the inputs $\left\{I_{l}\right\}$ are stochastically fed into the system continuously, the vector fields $\left\{f_{l}\right\}$ and iterated functions $\left\{g_{l}\right\}$ are stochastically switched [Gohara \& Okuyama, 1999b]. That is, there are two sets of dynamical systems (i.e. continuous and discrete dynamical systems, corresponding to the set $\left\{I_{l}\right\}$ ), and the former and latter systems are defined by the set $\left\{f_{l}\right\}$ and the set $\left\{g_{l}\right\}$, respectively. The set $\left\{g_{l}\right\}$ of the iterated function is named the Iterated Function System (IFS) [Barnsley, 1993]. Map $g_{l}$ is the contraction map when the discrete dynamics on the Poincaré section $\sum$ correspond to the random iteration algorithm using the IFS with probabilities [Gohara \& Okuyama, 1999b]. Similarly, the set $\left\{f_{l}\right\}$ is the vector field, called the Vector Fields System (VFS) [Gohara \& Okuyama, 1999a; Nishikawa \& Gohara, 2002]. Schematically, the relation among these sets is as follows:

$$
\left\{I_{l}\right\} \rightarrow\left\{f_{l}\right\} \rightarrow\left\{g_{l}\right\} .
$$

When a series of inputs $I(t)$ is rapidly switched with equal probability and is fed into a system to a sufficient extent, the set $C$ on the Poincaré section satisfies the following equation. It has been proven that this causes convergence of the attractive and unique invariant set $C$ [Barnsley, 1993].

$$
C=\bigcup_{l=1}^{L} g_{l}(C) .
$$

This set $C$ strongly affects the corresponding trajectory set $\Gamma(C)$ in the state space $\mathcal{M}$. Starting from the initial set $C$ in the space $\mathcal{M}$, the trajectory set $\Gamma(C)$ is the union of the trajectory set $\gamma_{l}(C)$ that is affected by each input $I(t)$, and it is also the set of the invariant trajectory, as follows:

$$
\Gamma(C)=\bigcup_{l=1}^{L} \gamma_{l}(C) .
$$

Equations (5) and (6) are proven analytically when all of the iterated functions $g_{l}(l=1,2, \ldots, L)$ are contractions. We can estimate the structure of the set $C$ or the trajectory set $\Gamma(C)$ using the correlation dimension value when input intervals (i.e. switching-time length) change. We can confirm the detailed theoretical explanation for the dynamical system developed by Gohara and Okuyama [1999a, 1999b], such as the nonautonomous system in which the input pattern is stochastically changed, and the empirical study of autonomous and nonautonomous systems for human movement conducted by Yamamoto and Gohara [2000]. If an excited attractor is assumed for human movement in this theory, continuous external input would generate movement patterns. Moreover, we can examine human motor control from the standpoint of a nonautonomous system on the condition that some movement patterns are excited stochastically. 


\section{Methods}

\subsection{Participants}

The participants were seven healthy right-handed men with no vision problems; handedness was determined by the Edinburgh Handedness Inventory. Their ages ranged between 28 and 39 years (mean age $\pm \mathrm{SD}=32.6 \pm 4.2$ years), their mean weight was $67.4 \pm 5.9 \mathrm{~kg}$, and their mean height was $171.7 \pm 2.7 \mathrm{~cm}$. They had no history of neurological or musculoskeletal disorders of the upper extremities. The procedure used in this study was approved by the Human Ethics Committee of Nanzan University, and all participants provided written informed consent before the study commenced.

\subsection{Experimental setting}

Four red light-emitting diodes (LEDs) (the size of one LED was $\phi 0.5 \mathrm{~cm}$ ) were grouped together at every third position under a $72 \times 50 \times 1 \mathrm{~cm}$ (width by depth by thickness) acrylic board (Fig. 2). One LED group (home position: $\mathrm{H}$ ) was located on the table $30 \mathrm{~cm}$ in front of the participants, and two LED groups were located $60 \mathrm{~cm}$ in front of them and
$30 \mathrm{~cm}$ from the center to the left and the right sides ( $\mathrm{L}$ and $\mathrm{R}$, respectively). Movement of a reflective marker $(1.5 \mathrm{~cm}$ in diameter $)$ pasted on the participants' index finger nail was sampled at $240 \mathrm{~Hz}$ by an optical motion-tracking system (Qualisys Oqus 300, Gothenburg, Sweden). Lighting of the LEDs was controlled with LabVIEW programming (NI CompactDAQ, NI9403). The presentation of stimuli was synchronized with Oqus.

\subsection{Task and procedure}

Participants put the tip of their index finger on $\mathrm{H}$ at the beginning of a measurement. When the left or right LED group was illuminated, which was accompanied by a sound (100 msec, $1000 \mathrm{~Hz})$, participants were supposed to point their index finger towards the appropriate LED group as rapidly as possible and then quickly return their finger to the $\mathrm{H}$ position. The light from the three LED groups was maintained for $400 \mathrm{msec}$, and 12 switchingtime lengths were set at $30,36,42,48,51,54$, $57,60,63,66,69$ and $72 \mathrm{bpm}$ (beats per minute). These bpm equal inter-beat intervals of 2.00, 1.67, $1.43,1.25,1.17,1.11,1.05,1.00,0.95,0.91,0.87$, and $0.83 \mathrm{sec}$ (seconds), respectively. Under the

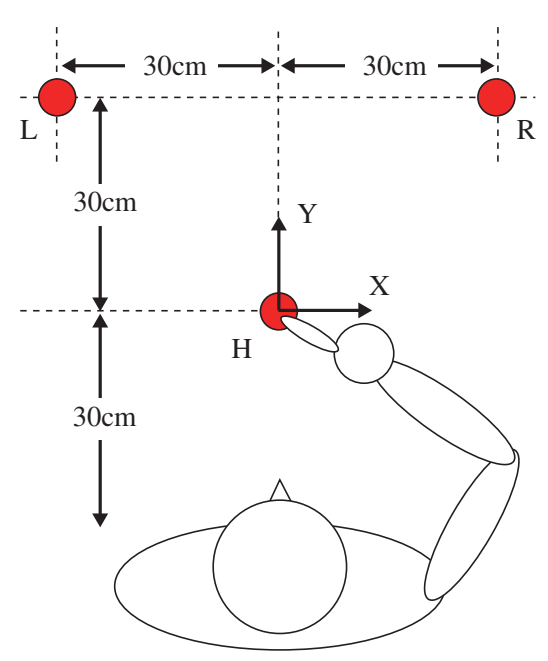

(a)

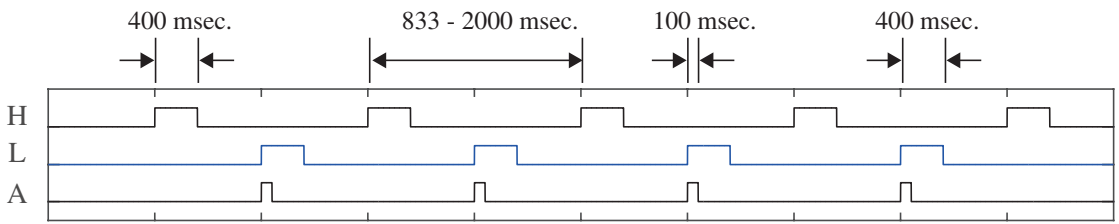

(b)

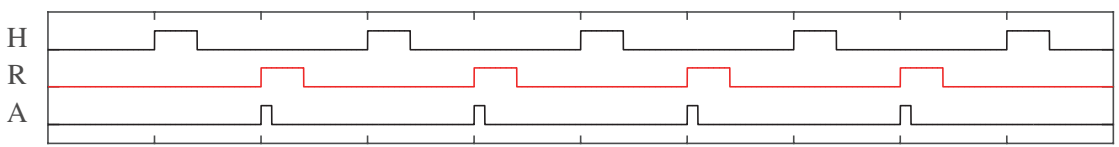

(c)

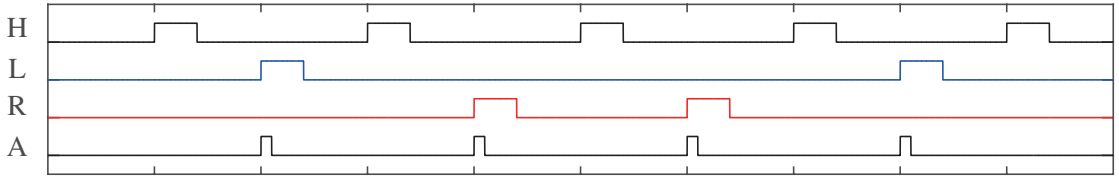

(d)

Fig. 2. Experimental setup. (a) Two target LED groups were located $60 \mathrm{~cm}$ in front of a participant and $30 \mathrm{~cm}$ from the center to both the left and right sides (L and R, respectively). Participants first placed their index finger on the LED group at home $(\mathrm{H})$, and they then moved their finger towards the LED group on the L or R that was illuminated randomly. They rapidly returned their finger to $\mathrm{H}$ after pointing to $\mathrm{L}$ or $\mathrm{R}$. Time-line diagrams: (b) the periodic input conditions for $\mathrm{L}$, (c) for $\mathrm{R}$, and (d) the switching input conditions. H, L, R, and A are the moments when the LEDs lit up at home and on the left and right sides and when the auditory signal sounded, respectively. 
periodic-input condition [the time-line diagram is shown in Figs. 2(b) and 2(c)], each participant repeatedly pointed at the same LED group (L or R) 64 times under each condition. Under the switchinginput condition [the time-line diagram is shown in Fig. 2(d)], they repeatedly pointed at the $\mathrm{L}$ or $\mathrm{R}$ LED group 512 times, and this sequence was randomized. For each switching-time length $T$, these pointing movements were divided into two trials under the periodic-input condition and into 16 trials under the switching-input condition to equalize the number of pointing times (32 times) and to avoid the influence of fatigue on the results. In total, 512 pointing movements included a thirdorder sequence effect denoting the following eight input pattern sequences: RRR, LRR, RLR, LLR, LRL, RRL, RLL, and LLL; this was similar to the tasks employed by Yamamoto and Gohara [2000] and Suzuki and Yamamoto [2015]. Data from the first two pointing movements in each trial were not analyzed because each trial consisted of a thirdorder sequence. Consequently, 480 pointing movements $(=30$ pointings $\times 16$ trials $=$ eight input patterns sequence $\times 60$ data points) were analyzed for each condition. Participants participated individually, and measurements were taken under up to three conditions per day. The experiment was divided into four or five days per participant. Participants could also take breaks as needed between trials. The duration of a break was determined by the participant according to his needs.

\subsection{Data analysis}

A right-handed orthogonal frame of reference was used, and the vectors $\mathrm{X}, \mathrm{Y}$, and $\mathrm{Z}$ defined its axes: $\mathrm{X}$ was horizontal and directed along the edge of the table in front of the participant, $\mathrm{Y}$ was perpendicular to the other two axes, and $\mathrm{Z}$ was vertical. The position data, which were based on a marker on the tip of the index finger, for the $\mathrm{X}$ and $\mathrm{Y}$ axes were calculated using Qualisys Track Manager (Gothenburg, Sweden). Data were smoothed with a second-order Butterworth digital low-pass filter using a $5-\mathrm{Hz}$ cut-off frequency. Data were differentiated to calculate velocities $\left(V_{x}\right.$ and $\left.V_{y}\right)$ on the $\mathrm{X}-\mathrm{Y}$ plane. Under all 12 conditions, the moment the LED group was lit at $H$ was at $\theta=0,2 \pi$ on the Poincaré section $(\Sigma)$ to embed the data in the state space $\mathcal{M}$. The correlation dimension $D_{c}$ for the set $g(C)$ on the Poincaré section $\Sigma(\theta=2 \pi)$ was estimated using the correlation integral calculated using the Grassberger-Procaccia (G-P) algorithm [Grassberger \& Procaccia, 1983]. A fitting curve was approximated by an inverse proportion and an exponential function to examine the relationship between switching-time length $T$ and the correlation dimension $D_{c}$. The validity and reliability of this statistical model (a fitting curve) were evaluated using Akaike's information criterion (AIC).

\section{Results}

\subsection{Periodic-input condition}

Figure 3 shows the results for participant 2 under the periodic-input condition $(0.83 \mathrm{sec}, 72 \mathrm{bpm})$ as a typical example. Figure 3(a) portrays the changes in $V x$ and $V y$ to exemplify the illumination of the left LED group under the periodic condition, and Fig. 3(b) shows when the right LED group was used. Poincaré section $\Sigma(\theta=0, \theta=2 \pi)$ is the moment the LED group was illuminated at $\mathrm{H}$. The set of trajectories in the state space $\mathcal{M}$ is expanded in Figs. 3(e) and 3(f) to depict the trajectories and the cross-section in the Poincaré section $\Sigma$. The trajectories in Figs. 3(e) and 3(f) correspond to Figs. 3(c) and 3(d), respectively. The trajectories in Figs. 3(e) and $3(\mathrm{f})$ began from the moment the LED group lit up at $\mathrm{H}$, the Poincaré section $\Sigma(\theta=0)$, until the next moment they lit up at $\mathrm{H}, \Sigma(\theta=2 \pi)$. The motion figures in the square box below Figs. 3(e) and $3(\mathrm{f})$ show the motion of the participants at each time point and at a time roughly equal to the pointing to each LED group. These results show that the set $\Gamma(C)$ of all trajectories starting from the initial set $C$ at $\theta=0$ on the Poincaré section, which is a discrete dynamical system, terminated at the initial set $C$ and again at $\theta=2 \pi$. The set $\gamma_{L}\left(C_{L}\right)$ of trajectories under the periodic condition when the LED group on the left was continuously lit starting from the initial set $C_{L}$ at $\theta=0$ terminated at the set $g_{L}\left(C_{L}\right)$ at $\theta=2 \pi$. The set $\gamma_{R}\left(C_{R}\right)$ of trajectories under the periodic condition when the LED group on the right was continuously lit starting from the initial set $C_{R}$ at $\theta=0$ terminated at the set $g_{R}\left(C_{R}\right)$ at $\theta=2 \pi$. Table 1 shows the results of a oneway multivariate analysis of variance (MANOVA) (Wilks' $\lambda$ ) for each participant and each periodicinput interval. These results reveal a significant difference between two clusters consisting of the set $\left[g_{L}\left(C_{L}\right), g_{R}\left(C_{R}\right)\right]$ on the Poincaré section $(\theta=2 \pi)$ and a significant difference in 82 of the 84 cases $(=$ seven participants $\times 12$ switching-time length 
Left LED lit

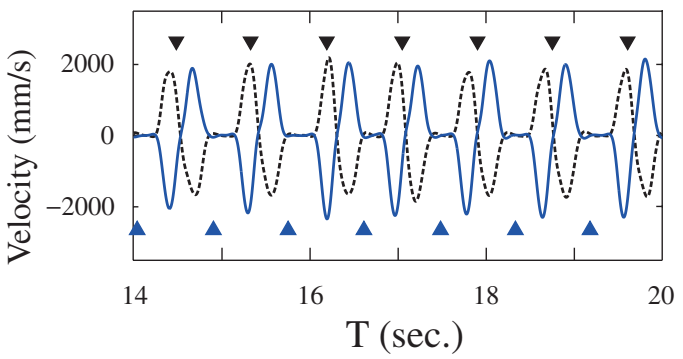

(a)

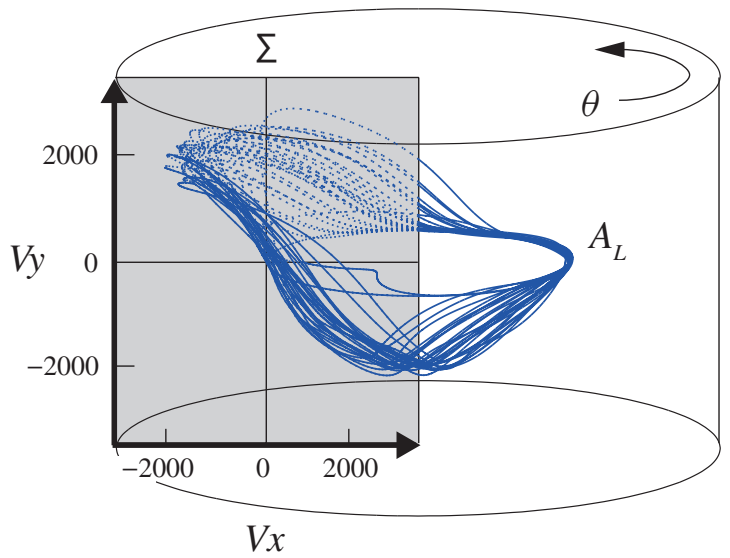

(c)

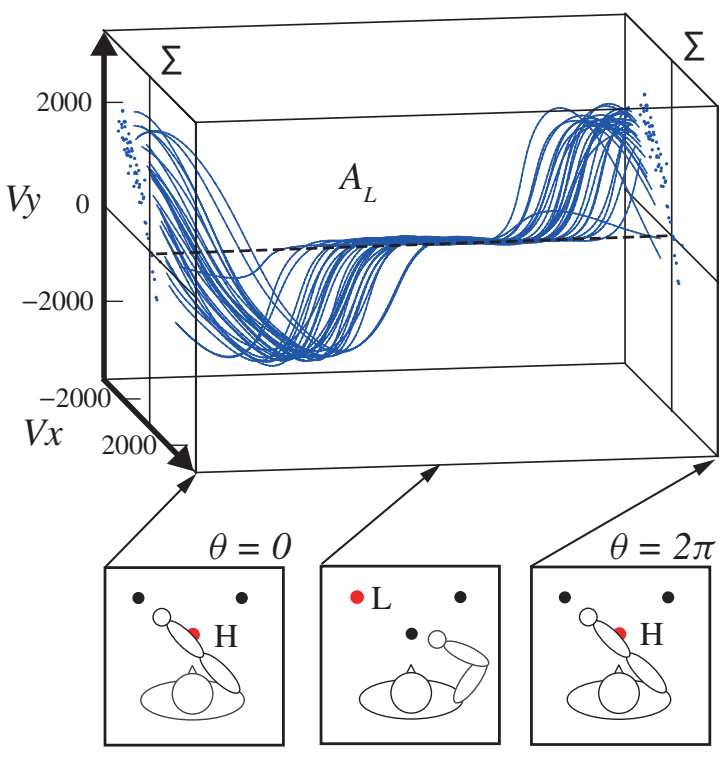

(e)

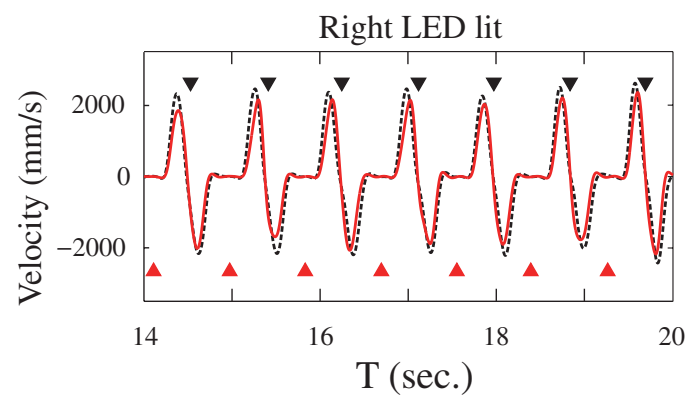

(b)

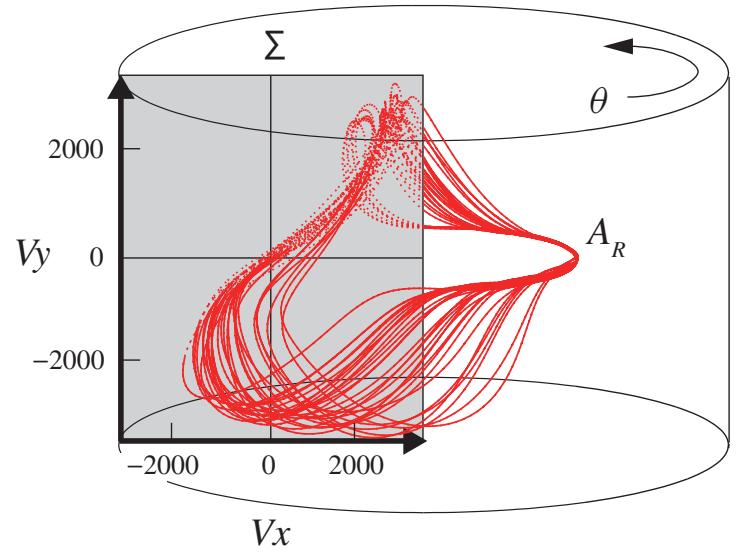

(d)

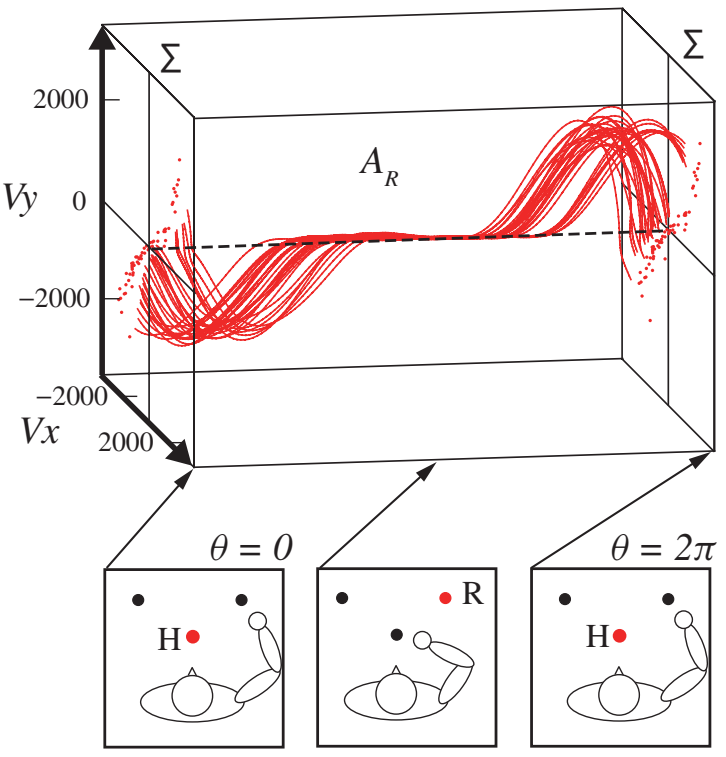

(f)

Fig. 3. A typical example of the periodic-input condition (participant 2, the switching-time length was 0.83 sec, 72 bpm). (a) and (b) are time-series data over a period of $6 \mathrm{sec}$ when the left (L) and the right (R) LED groups emitted repeatedly. The blue and red solid lines are the velocity $V x$ on the $\mathrm{X}$-axis, and the black dotted lines are the velocity $V y$ on the $\mathrm{Y}$-axis. The black, blue, and red triangles indicate the times when H, L, and R were lit, respectively. (c), (d), (e) and (f) present typical examples of the state space and the set $C$ on the Poincaré section. The set $\Gamma(C)$ of the trajectories starting from an initial state on $\Sigma(\theta=0)$ in the state space was drawn after cycling 30 times around the cylinder for each input in the examples. Additionally, the trajectories before and after that at $\theta=0$ and $2 \pi$ were omitted to confirm the set $C$ on the Poincaré section. 
Table 1. Results of MANOVA (Wilks' $\lambda$ ) of the differences between the mean left (L) and right (R) side input patterns on the Poincaré section $(\theta=2 \pi)$ under the periodic-input condition.

\begin{tabular}{cccccccccccccc}
\hline & \multicolumn{10}{c}{ Switching-Time Length (sec) } \\
\cline { 2 - 12 } Participant & 0.83 & 0.87 & 0.91 & 0.95 & 1.00 & 1.05 & 1.11 & 1.18 & 1.25 & 1.43 & 1.67 & 2.00 \\
\hline$\# 1$ & $0.15^{* *}$ & $0.34^{* *}$ & $0.38^{* *}$ & $0.08^{* *}$ & $0.05^{* *}$ & $0.18^{* *}$ & $0.29^{* *}$ & $0.13^{* *}$ & $0.26^{* *}$ & $0.10^{* *}$ & $0.01^{* *}$ & $0.86^{* *}$ \\
$\# 2$ & $0.51^{* *}$ & $0.77^{* *}$ & $0.83^{* *}$ & $0.89^{* *}$ & $0.31^{* *}$ & $0.02^{* *}$ & $0.10^{* *}$ & $0.10^{* *}$ & $0.03^{* *}$ & $0.22^{* *}$ & $0.13^{* *}$ & $0.41^{* *}$ \\
$\# 3$ & $0.54^{* *}$ & $0.43^{* *}$ & $0.37^{* *}$ & $0.11^{* *}$ & $0.21^{* *}$ & $0.13^{* *}$ & $0.13^{* *}$ & $0.08^{* *}$ & $0.02^{* *}$ & $0.01^{* *}$ & $0.08^{* *}$ & $0.71^{* *}$ \\
$\# 4$ & $0.80^{* *}$ & $0.52^{* *}$ & $0.14^{* *}$ & $0.40^{* *}$ & $0.21^{* *}$ & $0.19^{* *}$ & $0.17^{* *}$ & $0.20^{* *}$ & $0.10^{* *}$ & $0.07^{* *}$ & $0.22^{* *}$ & $0.29^{* *}$ \\
$\# 5$ & $0.83^{* *}$ & $0.64^{* *}$ & $0.38^{* *}$ & $0.49^{* *}$ & $0.07^{* *}$ & $0.04^{* *}$ & $0.02^{* *}$ & $0.08^{* *}$ & $0.05^{* *}$ & $0.03^{* *}$ & $0.03^{* *}$ & $0.53^{* *}$ \\
$\# 6$ & $0.33^{* *}$ & $0.32^{* *}$ & $0.50^{* *}$ & $0.69^{* *}$ & $0.87^{* *}$ & $0.63^{* *}$ & 0.96 & $0.53^{* *}$ & $0.11^{* *}$ & $0.06^{* *}$ & $0.24^{* *}$ & $0.49^{* *}$ \\
$\# 7$ & $0.08^{* *}$ & $0.09^{* *}$ & $0.04^{* *}$ & $0.28^{* *}$ & $0.04^{* *}$ & $0.37^{* *}$ & $0.09^{* *}$ & $0.16^{* *}$ & $0.51^{* *}$ & $0.57^{* *}$ & $0.65^{* *}$ & 0.98 \\
\hline
\end{tabular}

${ }^{* *} p<0.01$.

conditions $)(\approx 97.6 \%)$. As shown in the schematic diagrams in Figs. 1(a) and 1(b), there were two attractors of motion patterns $\left[A_{L}, A_{R}\right.$ in Figs. 3(c)$3(\mathrm{f})]$ inherent in the pointing movements towards the left and right sides, and these attractors were excited by external input and may be regarded as limit-cycle attractors.

\subsection{Switching-input condition}

Figure 4 shows the results for participant 2 under the switching-input condition (0.83 sec, $72 \mathrm{bpm})$ as a typical example. The blue and red trajectories in Fig. 4(a) show that the preceding inputs were left and right, respectively. Figures 4(b) and 4(c) show that the trajectories were embedded in the state space $\mathcal{M}$. Similar to the depiction in Fig. 3, the moment that the LED group lit up at the $\mathrm{H}$ position is located at $\theta=0$ and $\theta=2 \pi$ on the Poincaré section. The trajectories in the state space $\mathcal{M}$ are shown unfolded in Figs. 4(d) and 4(e). The blue lines in Figs. 4(b) and 4(d) show the results when the preceding input was $\mathrm{L}$ and the current input was $\mathrm{L}(\mathrm{LL})$; the red lines show the results when the preceding input was $\mathrm{R}$ and the current input was $\mathrm{L}$ (RL). The blue lines in Figs. 4(c) and 4(e) show the results when the preceding input was $L$ and the current input was $\mathrm{R}(\mathrm{LR})$; the red lines show the results when the preceding input was $\mathrm{R}$ and the current input was $\mathrm{R}(\mathrm{RR})$. The motion figures shown in the square boxes above and below Figs. 4(d) and 4(e) show the motion of the participants at each time point and at a time roughly equal to pointing to each LED group. Figure 4(d) shows that the set $\gamma_{L}\left(C_{L}\right)$ of trajectories after the left LED group was illuminated and the set $\gamma_{R}\left(C_{R}\right)$ of trajectories after the right LED group was illuminated starting from the initial sets $C_{L}$ and $C_{R}$ at $\theta=0$ terminated in the set $g_{L}\left(C_{L}\right)$ at $\theta=2 \pi$. Figure $4(\mathrm{e})$ shows that the set $\gamma_{L}\left(C_{L}\right)$ of trajectories when the left LED group was illuminated and the set $\gamma_{R}\left(C_{R}\right)$ of trajectories when the right LED group was illuminated starting from the initial set $C_{L}$ and $C_{R}$ at $\theta=0$ terminated in the set $g_{R}\left(C_{R}\right)$ at $\theta=2 \pi$. These results indicate that the continuous pointing movements towards the left and right sides followed an approximately fractallike structure.

A one-way MANOVA (Wilks' $\lambda$ ) was performed to test for the equality of the means of the multiple variables in the clusters of the set that were LL and $\mathrm{RL}$, and RR and LR, respectively, to examine the third-order sequence effect on the Poincaré section $(\theta=2 \pi)$ for each participant and each switchinginput condition (Table 2). Figure 5 shows typical results on the Poincaré section $(\theta=2 \pi)$ for each participant and the five selected switching-input conditions. The results reveal significant differences in 72 of the 84 cases (= seven participants $\times$ switching-time length 12 conditions) $(\approx 85.7 \%)$ for pointing movement switching towards the left side and in 53 of the 84 cases $(\approx 63.1 \%)$ for pointing movement switching towards the right side. This result indicates that not only an attractor of pointing movement towards the left and right directions existed, as revealed under the periodic-input condition, but also the preceding input and current input formed four clusters (LL, RL, RR, and LR) on the Poincaré section $\Sigma$ with a third-order sequence effect. Figure 6 shows the trajectories in an unfolded cylinder during the switching-input condition under three $(0.83 \mathrm{sec}, 0.95 \mathrm{sec}, 2.00 \mathrm{sec})$ of the 12 conditions selected as a typical example. The trajectories during the long-interval condition (2.00 sec, $30 \mathrm{bpm}$ ) seem to be simple trajectories while the sequence 
effect remained, because the trajectories seem to have converged closer to each other on the Poincaré section $(\theta=2 \pi)$ and do not appear to have spread on the section [Fig. 6(a)]. In fact, eight trajectories were generated by a third-order sequence effect as the switching-time length shortened [Figs. 6(b) and $6(\mathrm{c})$ ]. Consequently, these results indicate that these trajectories were affected by hysteresis (i.e. a sequence from the input before the preceding input to the current input).

Figure 7 shows an ellipse of equal probability of the set $C$ on the Poincaré section $(\theta=2 \pi)$. The maps of $\mathrm{L}$ and $\mathrm{R}$ in Fig. 7(a), the contents of which were influenced by the preceding inputs [shown in Fig. 7(b)], consist of the maps LL and $\mathrm{RL}$ and the maps RR and LR, respectively. Moreover, the maps LL, RL, RR, and LR in Fig. 7(b), the contents of which were influenced by the inputs before the preceding inputs [shown in Fig. 7(c)], consist of the maps LLL and RLL, LRL and RRL, RRR and LRR, and LLR and RLR, respectively. These relationships show that these maps on the Poincaré section show the third-order sequence effect, as we confirmed from the results of the oneway MANOVA analysis of the Poincaré maps under the switching-input conditions (shown in Table 2). These results imply that the variability during continuous movement with the switching input was not

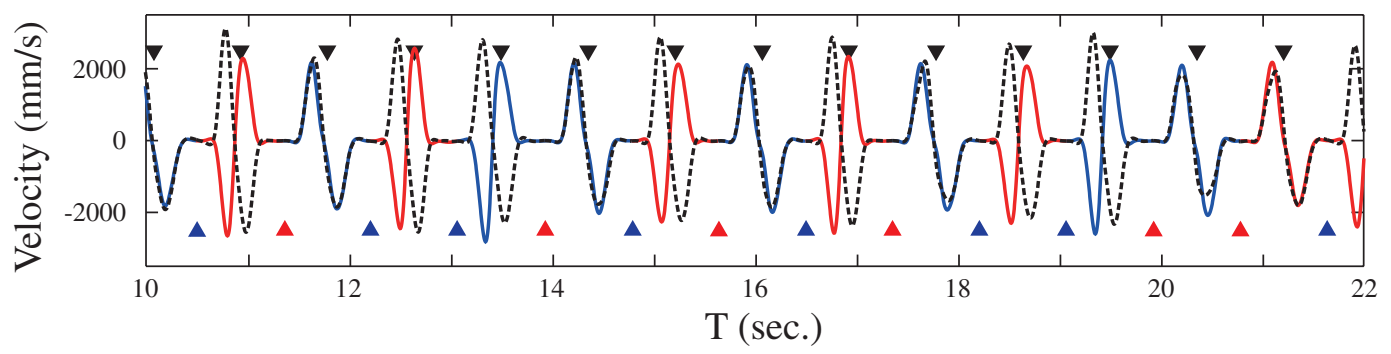

(a)

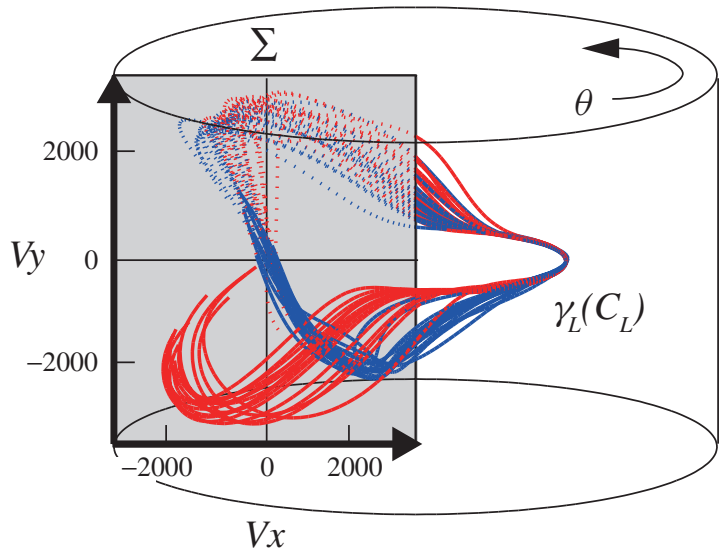

(b)

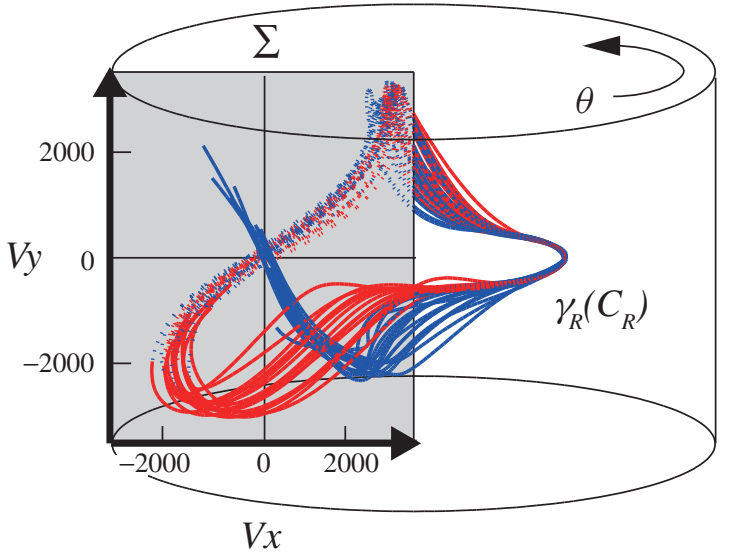

(c)

Fig. 4. A typical example under the switching-input condition (participant 2, input interval 0.83 sec, 72 bpm). (a) Time-series data for a period of $12 \mathrm{sec}$ during the switching-input condition. The black, blue, and red triangles indicate when the LEDs at home $(\mathrm{H})$, on the left side $(\mathrm{L})$, and on the right side (R) lit up, respectively. The blue and red solid lines are the velocity $V x$ on the $\mathrm{X}$-axis, and the black dotted line is the velocity $V y$ on the Y-axis. (b) and (c) The state space $\mathcal{M}$. (d) and (e) The unfolded state space. The blue and red lines indicate that the preceding inputs were $\mathrm{L}$ and $\mathrm{R}$, respectively. The blue line in (b) and (d) shows the results when the preceding input was L and the current input was L (LL); the red line shows the results when the preceding input was $\mathrm{R}$ and the current input was $\mathrm{L}(\mathrm{RL})$. The blue line in (c) and (e) shows the results when the preceding input was $\mathrm{L}$ and the current input was $\mathrm{R}(\mathrm{LR})$; the red line shows the results when the preceding input was $\mathrm{R}$ and the current input was $\mathrm{R}(\mathrm{RR})$. The set $g(C)$ on the Poincaré section $\theta=2 \pi$ (when the LED group at $\mathrm{H}$ was activated) is represented by the same color as each trajectory. The corresponding small illustrations at the top and bottom in (d) and (e) show the motion of participants at each point and approximately when pointing to each LED. The blue and red illustrations depict the motion pattern in which the preceding inputs were $\mathrm{L}$ and $\mathrm{R}$, respectively. Additionally, the trajectories before and after that at $\theta=0$ and $2 \pi$ were omitted to confirm the set $g(C)$ on Poincaré section. 


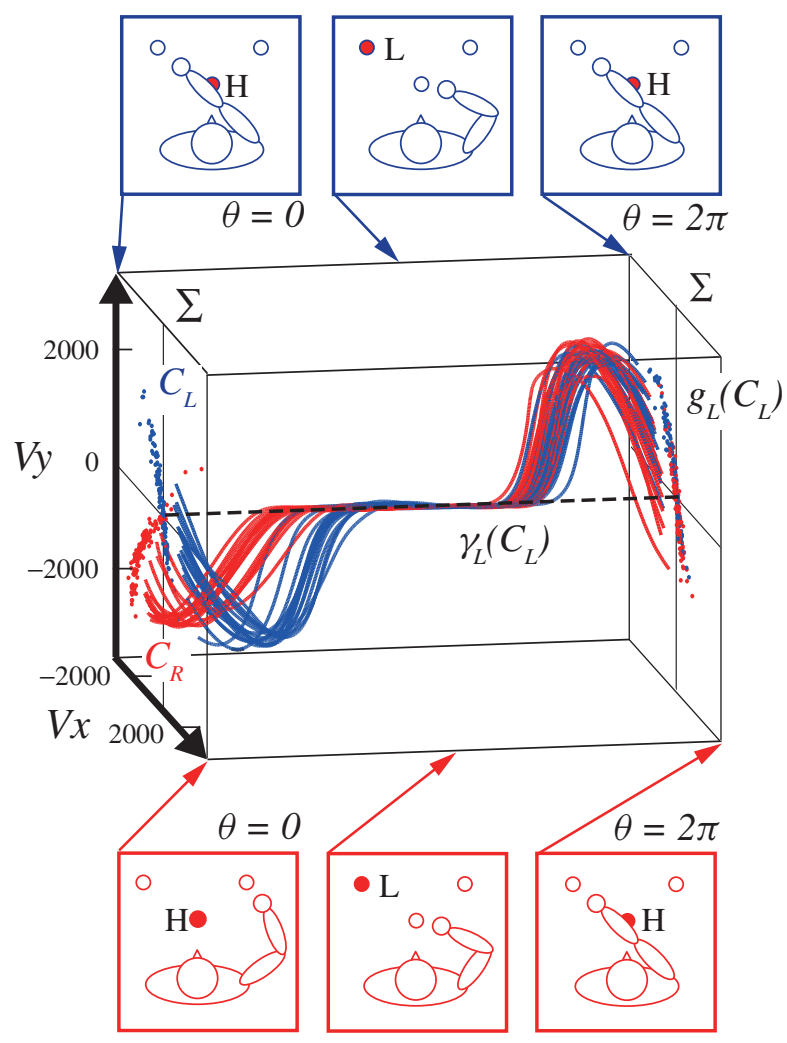

(d)

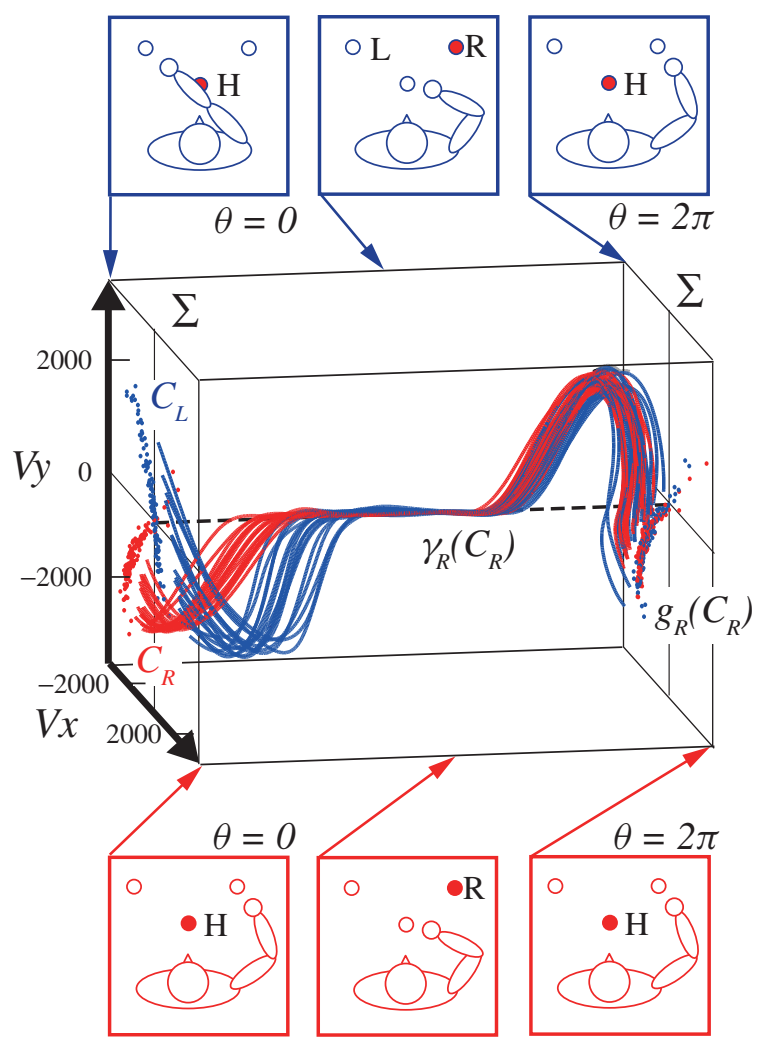

(e)

Fig. 4. (Continued)

Table 2. Results of MANOVA (Wilks' $\lambda$ ) of differences between the mean left side-left side (LL) and right side-left side $(\mathrm{RL})$, the mean right side-right side (RR) and left side-right side (LR) input pattern on the Poincaré section $(\theta=2 \pi)$ under the switching-input condition.

\begin{tabular}{|c|c|c|c|c|c|c|c|c|c|c|c|c|c|}
\hline \multirow[b]{2}{*}{ Participant } & & \multicolumn{12}{|c|}{ Switching-Time Length (sec) } \\
\hline & & 0.83 & 0.87 & 0.91 & 0.95 & 1.00 & 1.05 & 1.11 & 1.18 & 1.25 & 1.43 & 1.67 & 2.00 \\
\hline \multirow[t]{2}{*}{$\# 1$} & $\mathrm{LL} / \mathrm{RL}$ & $0.91^{* *}$ & $0.96^{*}$ & $0.77^{* *}$ & $0.80^{* *}$ & $0.87^{* *}$ & $0.76^{* *}$ & $0.63^{* *}$ & $0.83^{* *}$ & $0.78^{* *}$ & $0.63^{* *}$ & $0.92^{* *}$ & $0.89^{* *}$ \\
\hline & RR/LR & $0.81^{* *}$ & 0.98 & $0.77^{* *}$ & $0.91^{* *}$ & $0.88^{* *}$ & $0.79^{* *}$ & $0.94^{* *}$ & 0.99 & $0.92^{* *}$ & 0.99 & 0.98 & $0.96^{*}$ \\
\hline \multirow[t]{2}{*}{$\# 2$} & $\mathrm{LL} / \mathrm{RL}$ & $0.86^{* *}$ & $0.63^{* *}$ & $0.85^{* *}$ & $0.62^{* *}$ & $0.80^{* *}$ & $0.75^{* *}$ & $0.75^{* *}$ & $0.83^{* *}$ & $0.96^{* *}$ & $0.93^{* *}$ & 1.00 & 0.98 \\
\hline & $\mathrm{RR} / \mathrm{LR}$ & 0.99 & 0.99 & 0.98 & $0.97^{*}$ & 0.99 & $0.92^{* *}$ & $0.95^{* *}$ & $0.92^{* *}$ & $0.97^{*}$ & 0.98 & 0.98 & 0.99 \\
\hline \multirow{2}{*}{$\# 3$} & $\mathrm{LL} / \mathrm{RL}$ & $0.70^{* *}$ & $0.96^{* *}$ & 0.99 & $0.96^{* *}$ & $0.97^{*}$ & $0.86^{* *}$ & $0.92^{* *}$ & $0.95^{* *}$ & $0.87^{* *}$ & $0.88^{* *}$ & $0.87^{* *}$ & $0.85^{* *}$ \\
\hline & RR/LR & $0.66^{* *}$ & $0.75^{* *}$ & $0.81^{* *}$ & $0.90^{* *}$ & $0.92^{* *}$ & $0.96^{* *}$ & 0.98 & $0.97^{*}$ & $0.95^{* *}$ & $0.93^{* *}$ & $0.96^{*}$ & 0.99 \\
\hline \multirow[t]{2}{*}{$\# 4$} & $\mathrm{LL} / \mathrm{RL}$ & $0.88^{* *}$ & $0.91^{* *}$ & 0.98 & $0.93^{* *}$ & $0.94^{* *}$ & $0.96^{* *}$ & $0.92^{* *}$ & $0.94^{* *}$ & $0.96^{* *}$ & $0.97^{*}$ & 0.98 & 0.99 \\
\hline & $\mathrm{RR} / \mathrm{LR}$ & $0.81^{* *}$ & $0.91^{* *}$ & $0.97^{*}$ & $0.93^{* *}$ & 1.00 & 1.00 & 0.99 & 0.99 & 0.99 & 1.00 & $0.97^{*}$ & 0.99 \\
\hline \multirow[t]{2}{*}{$\# 5$} & $\mathrm{LL} / \mathrm{RL}$ & $0.97^{*}$ & 0.98 & 0.98 & $0.95^{* *}$ & $0.95^{* *}$ & 0.99 & 0.99 & $0.88^{* *}$ & $0.89^{* *}$ & $0.86^{* *}$ & $0.96^{*}$ & $0.98^{*}$ \\
\hline & $\mathrm{RR} / \mathrm{LR}$ & $0.87^{* *}$ & $0.87^{* *}$ & $0.92^{* *}$ & $0.86^{* *}$ & $0.91^{* *}$ & $0.96^{* *}$ & $0.94^{* *}$ & $0.89^{* *}$ & $0.84^{* *}$ & $0.91^{* *}$ & $0.96^{* *}$ & $0.93^{* *}$ \\
\hline \multirow[t]{2}{*}{$\# 6$} & $\mathrm{LL} / \mathrm{RL}$ & $0.83^{* *}$ & $0.75^{* *}$ & $0.84^{* *}$ & $0.72^{* *}$ & $0.72^{* *}$ & $0.73^{* *}$ & $0.67^{* *}$ & $0.65^{* *}$ & $0.86^{* *}$ & $0.97^{*}$ & $0.97^{*}$ & 0.99 \\
\hline & $\mathrm{RR} / \mathrm{LR}$ & 0.99 & $0.90^{* *}$ & 0.99 & 0.99 & $0.86^{* *}$ & $0.87^{* *}$ & $0.85^{* *}$ & 0.98 & 0.99 & 1.00 & 0.98 & $0.94^{* *}$ \\
\hline \multirow[t]{2}{*}{$\# 7$} & $\mathrm{LL} / \mathrm{RL}$ & $0.96^{*}$ & $0.86^{* *}$ & $0.74^{* *}$ & $0.82^{* *}$ & $0.88^{* *}$ & $0.89^{* *}$ & $0.90^{* *}$ & $0.97^{*}$ & 1.00 & $0.94^{* *}$ & $0.91^{* *}$ & $0.97^{*}$ \\
\hline & RR/LR & 0.98 & $0.91^{* *}$ & $0.96^{* *}$ & 0.99 & $0.98^{*}$ & $0.97^{*}$ & $0.88^{* *}$ & 0.99 & $0.96^{*}$ & $0.96^{* *}$ & 1.00 & $0.97^{*}$ \\
\hline
\end{tabular}

${ }^{*} p<0.05,{ }^{* *} p<0.01$. 

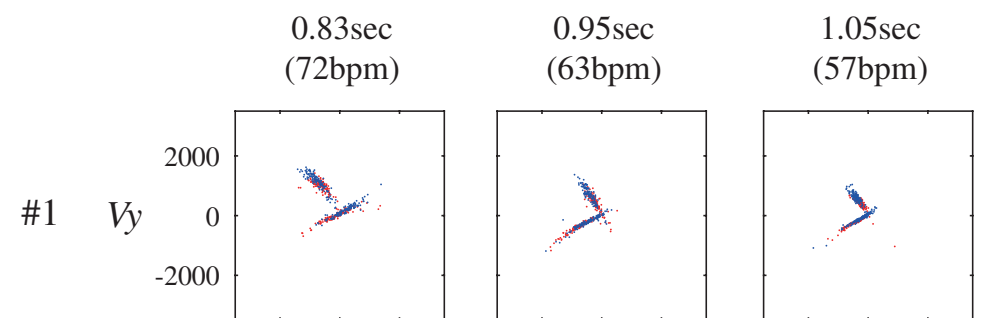

$1.43 \mathrm{sec}$

$2.00 \mathrm{sec}$

(42bpm)

(30bpm)
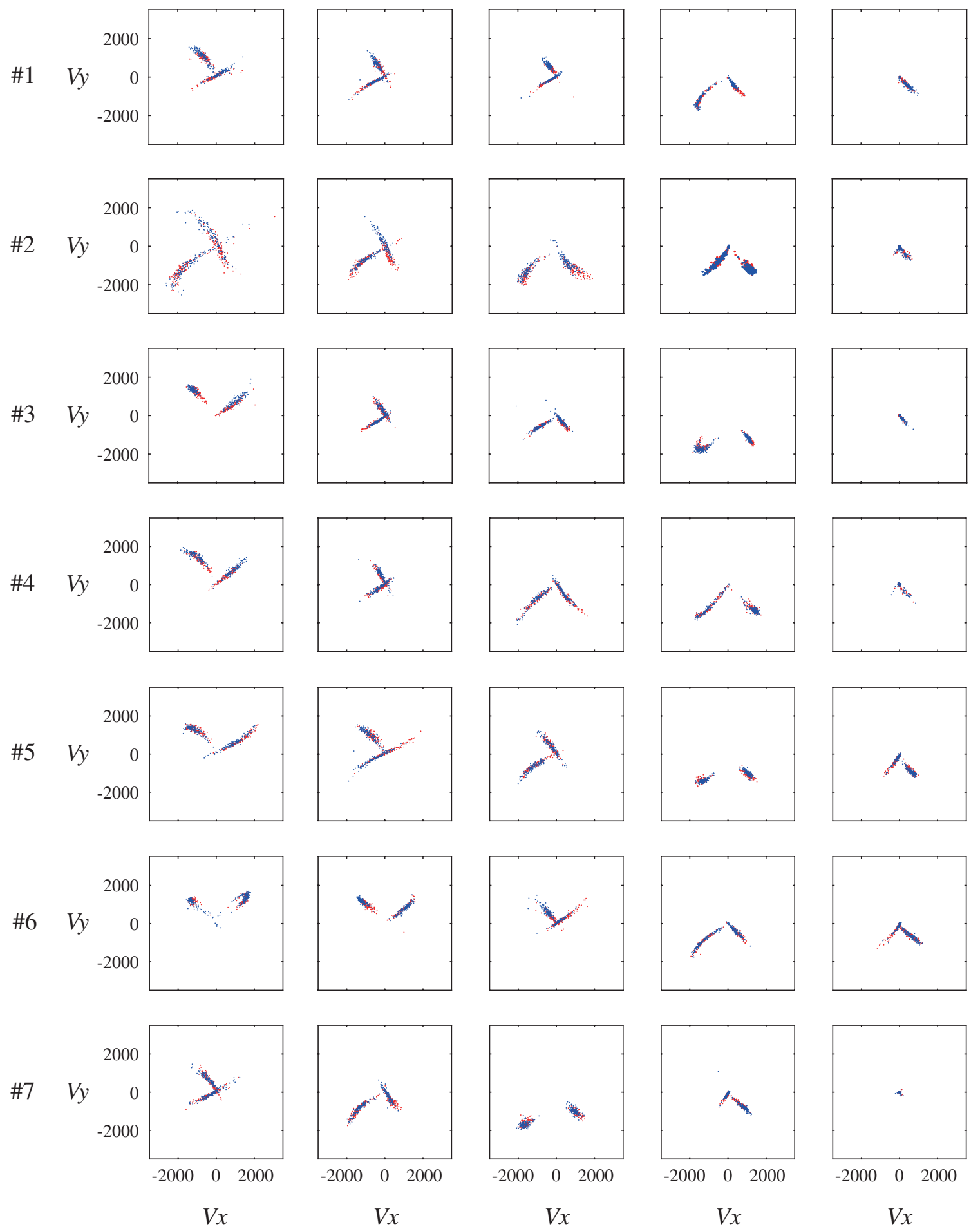

Fig. 5. Results of $g(C)$ on the Poincaré section $\sum(\theta=2 \pi)$ under five selected conditions $(0.83,0.95,1.05,1.43,2.00$ sec $)$ over all 12 conditions. The results from participants \#1 to \#7 are arranged in a row, and the five conditions are in columns. The blue and red dots indicate that the preceding inputs were $\mathrm{L}$ and $\mathrm{R}$, respectively. 


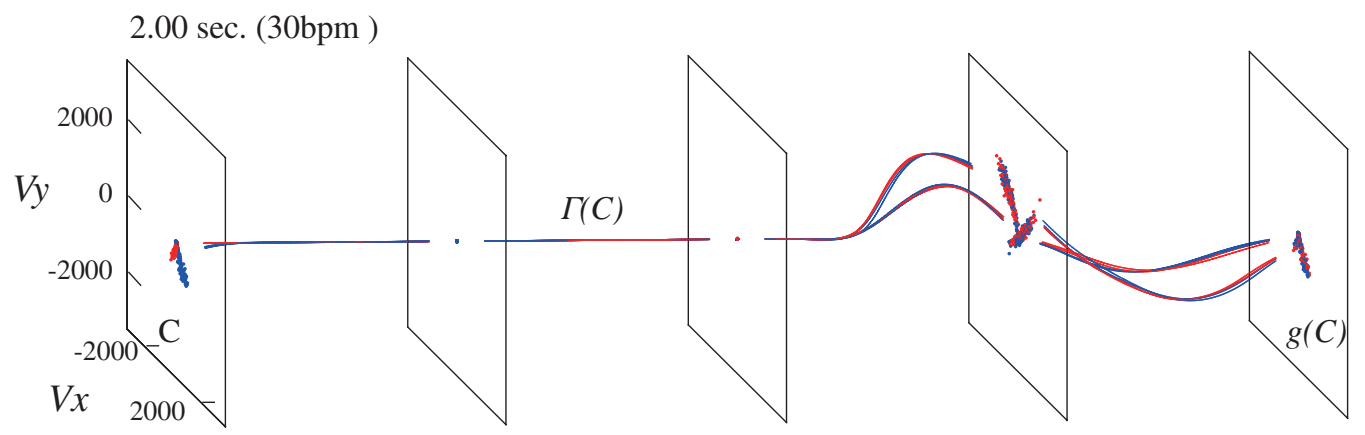

(a)

$0.95 \mathrm{sec} .(63 \mathrm{bpm})$

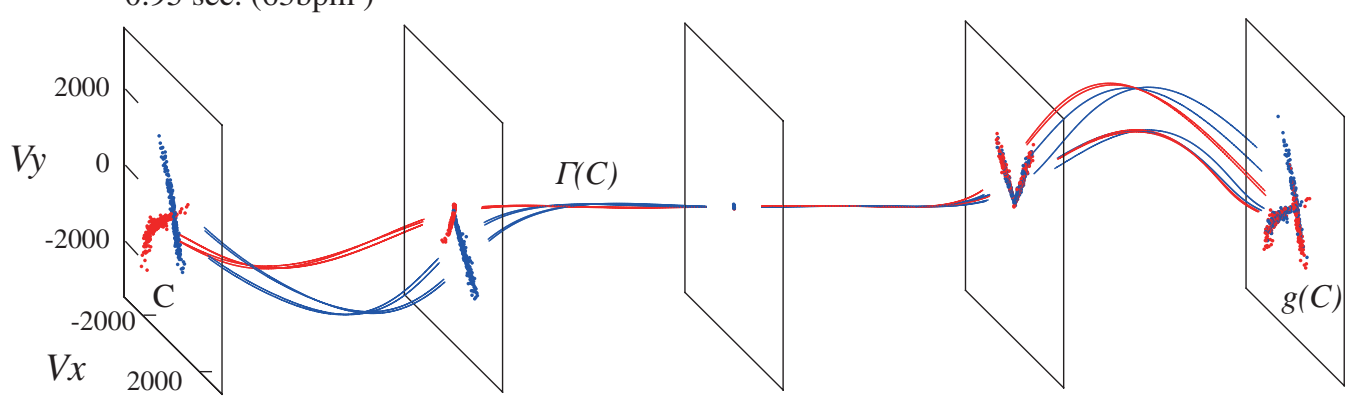

(b)

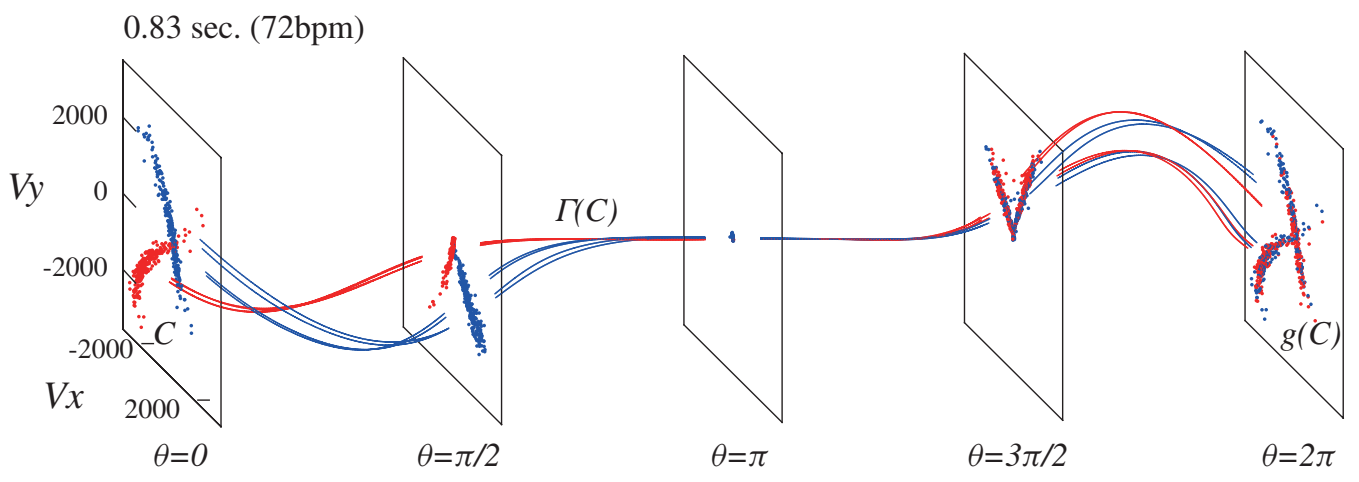

(c)

Fig. 6. The unfolded state space during the three switching-input conditions selected from all 12 conditions (data from participant 2). Data on the Poincaré section $\Sigma$ were obtained from participant 2 (480 data points for each section), and each trajectory depicts the mean trajectory of the set $\Gamma(C)$ of the third-order sequence. Additionally, the trajectories $\gamma(C)$ before and after those at $\theta=0, \pi / 2, \pi, 3 \pi / 2$, and $2 \pi$ were omitted to confirm the set $g(C)$ on the Poincaré section. 


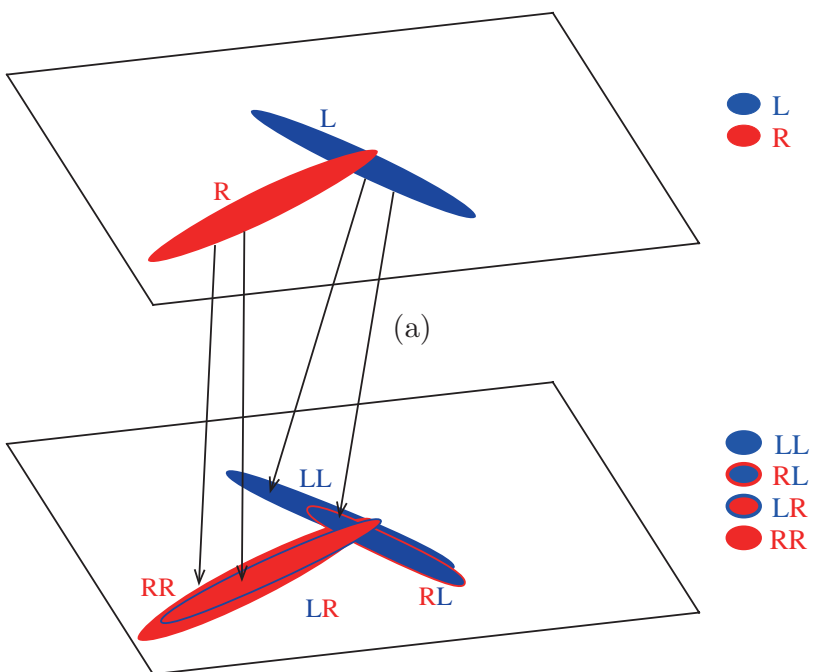

(b)

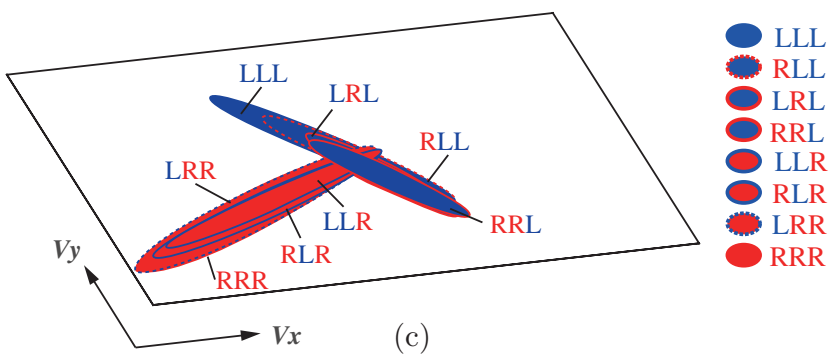

Fig. 7. The structure, which was affected by the third-order sequential effects, of the set $g(C)$ (an ellipse of equal probability) on the Poincaré section. (a) The result of the map during the current input, (b) the ellipses calculated when classified by the sequence of the preceding input and (c) the ellipses calculated when classified by the sequence of the input before the preceding input.

a stochastic distribution, such as a Gaussian distribution, random variation, a uniform distribution, or white noise, and that the variability is generated regularly by the fractal-like structure when a human switches rapidly between two movement patterns with external inputs.

\subsection{Correlation dimensions on Poincaré section}

Figure 8 is a typical example (participant 2) of the correlation integral $O(r)$ calculated by the $\mathrm{G}-\mathrm{P}$ method [Grassberger \& Procaccia, 1983] to quantify the set $g(C)$ on the Poincaré section under the switching-input condition. The correlation integral is the calculation used most widely for estimating the fractal dimension of small datasets, and the degree of incline in the log-log plot of Fig. 8 indicates the correlation dimension value. Figure $8(\mathrm{a})$ shows the results of the correlation integral for all 12 conditions; a part of Fig. 8(a) is expanded in Fig. 8(b). The inclination of the slope under each condition (i.e. the correlation dimension $D_{c}$ ) increased as an approximate function of decreases in the input interval.

Figure 9(a) shows a typical example of the set $g(C)$ on the Poincaré section $(\theta=2 \pi)$ under three selected conditions ( $0.83 \mathrm{sec}, 0.95 \mathrm{sec}, 2.00 \mathrm{sec})$ over all 12 conditions. Comparison of the set $g(C)$ during the longer switching-time length with the set $g(C)$ during the shorter switching-time length revealed that the former converged on the local region during the longer input interval condition (e.g. $2.00 \mathrm{sec}$, $30 \mathrm{bpm}$ ) and that the latter spread over the whole section during the shorter input interval condition (e.g. $0.83 \mathrm{sec}, 72 \mathrm{bpm}$ ). Consequently, the dimension shifted towards zero as the time interval increased, and it shifted to near two as the time interval decreased. This implies that the trajectories $\Gamma(C)$ and the set $g(C)$ gradually spread over the whole space as the input interval shortened. That is, the correlation dimension was nearly zero at the limit $T \rightarrow \infty$ because the trajectories seem to converge at a point in spite of having the same structure. On the other hand, the correlation dimension increased to near two at the limit $T \rightarrow 0$ because the trajectories spread around two attractors due to the rapid transitions between them [Nishikawa \& Gohara, 2002].

The upper part of Fig. 9(b) is the mean and the standard deviation of the correlation dimension for each of the switching-time lengths $T$. At first, the AIC was calculated using the correlation dimension of all participants under 12 conditions (84 data points $=$ seven participants $\times 12$ conditions) after fitting an inverse proportion curve with and without a constant and an exponential function. The leftmost green (inverse proportion without the constant), blue (inverse proportion with the constant), and red (exponential function) blank circles at the bottom of Fig. 9(b) present the results of this calculation. Next, we calculated the AIC for each fitting curve using 77 data points (= seven participants $\times 11$ conditions) after excluding only seven data points for the shortest input interval $(0.83 \mathrm{sec})$. The results are represented by the second leftmost blank circles. We repeatedly calculated the AIC after excluding the results of the correlation dimension from the shorter input interval conditions. The rightmost blank circles are the results of the AIC 


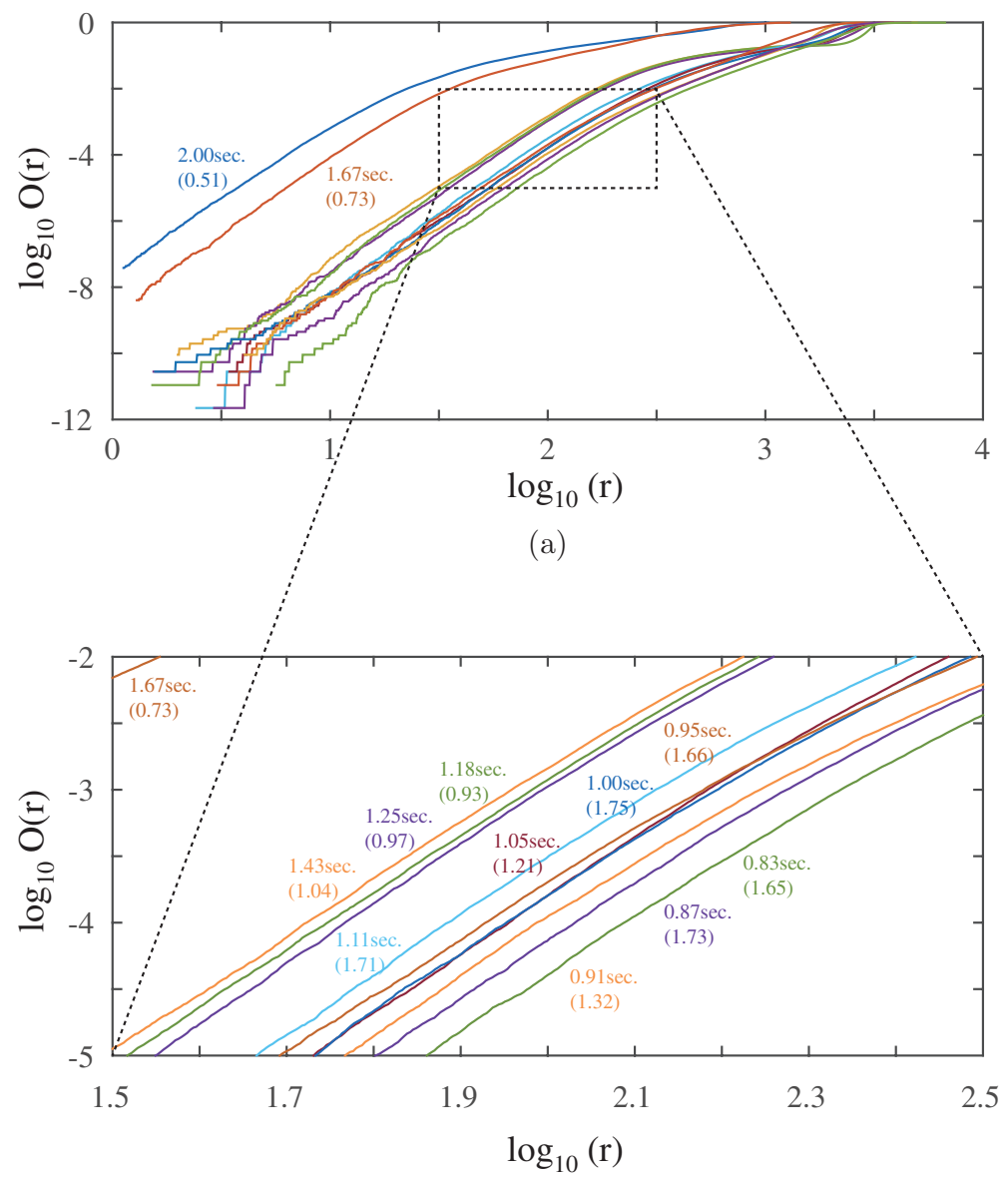

(b)

Fig. 8. Results of the correlation dimension from the correlation integral under all 12 switching-input conditions (data from participant 2). This is a typical example of how to calculate the set $g(C)$ on the Poincaré section $(\theta=2 \pi)$ with the correlation integral $O(r)$. (a) The results under all 12 switching-input conditions and (b) part of (a) that was magnified to confirm the slope in detail. In these figures, the line color is the same as the text color depicting each switching-time length $T$. The values in parentheses are the correlation dimension value. 


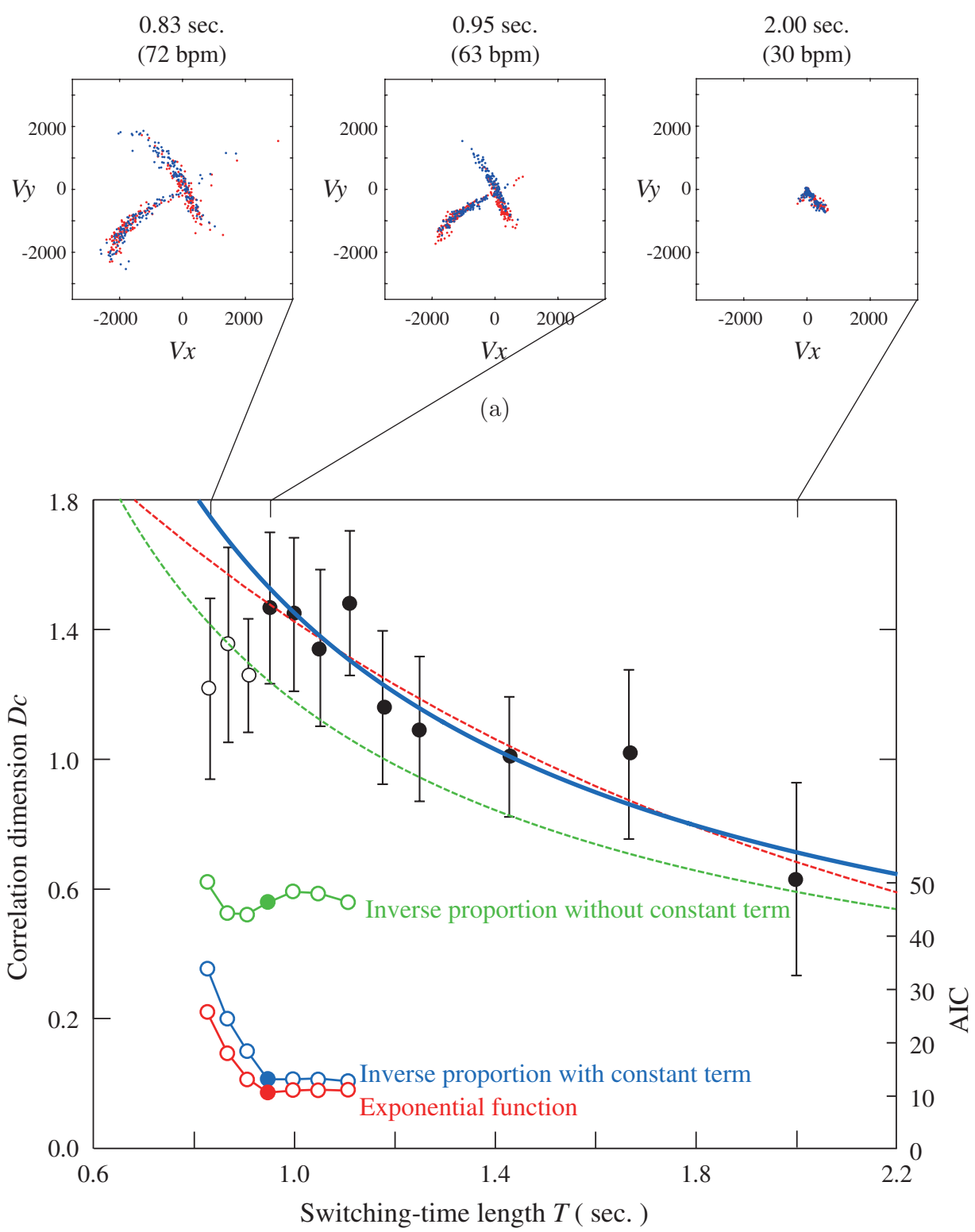

(b)

Fig. 9. Means and standard deviations of the correlation dimensions under all switching-time lengths $T$. (a) The set $g(C)$ on the Poincaré section $(\theta=2 \pi)$ for the typical example (participant 2) and (b) means (black circles) and standard deviations (error bars) calculated from the data obtained from all participants under all 12 conditions. The red dotted line, blue line, and green dotted line are the fitting curves with the exponential function and the inverse proportion with and without the constant term, respectively. A number of conditions were selected and analyzed based on the results for the AIC to plot these fitting curves. The results for the AIC are shown in the lower part of (b). The black circles in the upper part of (b) (nine conditions from $2.00 \mathrm{sec}$ in which the maximal switching-time length $T$ was $0.95 \mathrm{sec}$ ) were finally selected to fit the curve.

under only six conditions (42 data points). The AIC calculated for the first nine conditions (63 data points) from the longest input interval $(2.00 \mathrm{sec})$ to the ninth condition $(0.95 \mathrm{sec})$ differed from that calculated for the tenth, 11th, and 12th conditions due to the fitting curve (an inverse proportion with the constant term and an exponential function). The fitting curves for the nine conditions $(2.00 \mathrm{sec}$ to
$0.95 \mathrm{sec}$ ) appear in the upper part of Fig. 9(b). We selected the inverse proportion with the constant rather than the exponential function following the theoretical model developed by Gohara and Okuyama [1999b]. For a continuous switching pointing movement, the correlation dimension $D_{c}$ of the set $g(C)$ increased as the input interval decreased (e.g. $D_{c}=1.43 / T+0.002$ ). 


\section{Discussion}

Recent research has identified a fractal-like or selfsimilar structure not only in various physical phenomena [Arecchi et al., 1986; Constantin et al., 1991; Maas et al., 1997; Matias et al., 1997; Mestl et al., 1997; Schmiegel \& Eckhardt, 1997; Tanii et al., 1991, 1999] but also in continuous movements under human motor control for a particular input interval [Yamamoto \& Gohara, 2000] or for certain switching-time lengths $T$ [Suzuki \& Yamamoto, 2015]. The purpose of this study was to examine the regularity of the fluctuations in human motor control, specifically abrupt switching behavior with an external input, applying a theoretical model containing a function for switching-time length $T$ and self-similar structure to a continuous human pointing movement.

According to the theory of dynamical systems with external inputs, an attractor forms a movement pattern during a periodic-input condition [Gohara \& Okuyama, 1999a]. It has been confirmed that each movement pattern (i.e. the pointing movement towards the left and right diagonal directions in this study) forms limit-cycle attractors by a quantitative (Table 1) and geometric (Fig. 3) structure. In addition, the set $g(C)$ on the Poincaré section was a noninteger value for switching-input conditions. If a correlation dimension is a noninteger value, the geometric structure is known as a fractal-like structure [Grassberger \& Procaccia, 1983; Suzuki \& Yamamoto, 2015]. In other words, these results show that in this study the variability in human motor control, especially pointing movements towards the left and right diagonal forward directions, had a fractal-like or self-similar structure. This implies that the noise in a movement pattern, which is generated via motor control in humans, is a structural fluctuation rather than stochastic noise. This means that four kinds of movement pattern were generated regularly and are affected by the third-order effect, which corresponds to the sequence from the input pattern before the preceding input pattern to that from the current input pattern. In other words, human movement is characterized by the sequence of the input patterns, which is the same as the changes of motor frequency in the hysteresis effect [Kostrubiec et al., 2006; Wallace, 1996; Zanone et al., 2010]. This suggests that the dynamical system perspective reveals the common underlying characteristics and patterns like self-similar structures or hysteresis in human behavior.

Furthermore, these noninteger values differed according to switching-time length. The results obtained from the fitting curve with an inverse proportion and an exponential function indicate that the relationship between the correlation dimension and the switching-time length was one of inverse proportion, which is the regular relationship. As Nishikawa and Gohara [2002] suggested, the T (time)-dependence of the correlation dimension can be understood by intuition, as the dynamics at both limits, $T \rightarrow 0$ and $T \rightarrow 2$, of the curve are considered. That is, two attractors converged near the region at $T \rightarrow \infty$ [the sets on the Poincaré section at $2.00 \mathrm{sec}$ in Fig. 5 rightmost in column, Figs. 6(a) and 9 (a) rightmost], and the correlation dimension approached zero. In contrast, the trajectories became disordered around the two attractors, with a rapid transition at $T \rightarrow 0$, and the correlation dimension approached two [Nishikawa \& Gohara, 2002 .

The relationship between the switching-time length $T$ and the correlation dimension $D_{c}$ during continuous pointing movement is also expected to be one of inverse proportion for human motor control, as in the theoretical model developed by Gohara and Okuyama [1999b] and the empirical study involving an experiment with an electrical circuit conducted by Nishikawa and Gohara [2002]. That is, this relationship is approximately $D_{c} \propto$ $1 / T$. The experimental data collected by Suzuki and Yamamoto [2015] showed that changes between skill levels in table tennis differed in the correlation dimension, but the maximum of the correlation dimension remained the same $\left(D_{c} \approx 1.8\right)$. In this study, the ceiling value of the correlation dimension was 1.4 or 1.5 for human movement relating to abrupt switching of pointing direction as the switching-time length decreased. Therefore, the correlation dimension in human motor control does not endlessly proceed upward in inverse proportion to the switching-time length [Fig. 9(b)] and the variability does not become completely random and may be a specific noninteger value, such as 1.4 or 1.5 in the pointing movement and 1.8 in table tennis [Suzuki \& Yamamoto, 2015]. This implies that the switching behavior between two attractors generates a fractal-like state in the set $g(C)$ on the Poincaré section and that the structure is 
self-similar or fractal-like rather than consisting of a stochastic error. This phenomenon shows that the switching-time length affects the complexity of human movement and that human behavior does not increase randomly as the switching-time length decreases. Finally, the result of the curve fitting was an inverse proportion with a constant term. The implication of this constant term in the switching behavior of two attractors suggests that human movement patterns are not uniform and are variable at only that rate (i.e. the value of the constant term). This also shows that human movement does not become completely random, as the correlation dimension values did not exceed 1.4 or 1.5 in Fig. 9(b).

\section{Acknowledgment}

This work was supported by JSPS KAKENHI 20240060, 24240085, and 24700629.

\section{References}

Arecchi, F. T., Gadomski, W. \& Meucci, R. [1986] "Generation of chaotic dynamics by feedback on a laser," Phys. Rev. A 34, 1617-1620.

Barnsley, M. [1993] Fractals Everywhere (Academic Press, Boston).

Constantin, P., Procaccia, I. \& Sreenivasan, K. R. [1991] "Fractal geometry of isoscalar surfaces in turbulence: Theory and experiments," Phys. Rev. Lett. 67, 17391742.

Ding, M., Chen, Y. \& Kelso, J. A. S. [2002] "Statistical analysis of timing errors," Brain Cogn. 48, 98106.

Gohara, K. \& Okuyama, A. [1999a] "Dynamical systems excited by temporal inputs: Fractal transition between excited attractors," Fractals 7, 205-220.

Gohara, K. \& Okuyama, A. [1999b] "Fractal transition: Hierarchical structure and noise effect," Fractals $\mathbf{7}$, 313-326.

Grassberger, P. \& Procaccia, I. [1983] "Characterization of strange attractors," Phys. Rev. Lett. 50, 346349.

Haken, H., Kelso, J. A. S. \& Bunz, H. [1985] "A theoretical model of phase transitions in human hand movement," Biol. Cybern. 51, 347-356.

Harbourne, R. T. \& Stergiou, N. [2009] "Movement variability and the use of nonlinear tools: Principles to guide physical therapist practice," Phys. Ther. 89, 267-282.

Hausdorff, J. M. [2007] "Gait dynamics, fractals and falls: Finding meaning in the stride-to-stride fluctuations of human walking," Hum. Mov. Sci. 26, $555-589$.

Kelso, J. A. S. [1984] "Phase transitions and critical behavior in human bimanual coordination," Am. J. Physiol. 240, R1000-R1004.

Kijima, A., Yokoyama, K., Shima, H. \& Yamamoto, Y. [2014] "Emergence of self-similarity in football dynamics," Eur. Phys. J. B 87, 41.

Kirchner, M., Schubert, P., Liebherr, M. \& Haas, C. T. [2014] "Detrended fluctuation analysis and adaptive fractal analysis of stride time data in Parkinson's disease: Stitching together short gait trials," PLoS ONE 9, e85787.

Kostrubiec, V., Tallet, J. \& Zanone, P. [2006] "How a new behavioral pattern is stabilized with learning determines its persistence and flexibility in memory," Exp. Brain Res. 170, 238-244.

Maas, L. R. M., Benielli, D., Sommeria, J. \& Lam, F. P. A. [1997] "Observation of an internal wave attractor in a confined, stably stratified fluid," Nature $\mathbf{3 8 8}$, $557-561$.

Mandelbrot, B. B. [1967] "How long is the coast of Britain? Statistical self-similarity and fractal dimension," Science 156, 636-638.

Matias, M. A., Muñuzuri, V. P., Lorenzo, M. N., Mariño, I. P. \& Villar, P. [1997] "Observation of a fast rotating wave in rings of coupled chaotic oscillators," Phys. Rev. Lett. 78, 219-222.

Mestl, T., Bagley, R. J. \& Glass, L. [1997] "Common chaos in arbitrarily complex feedback networks," Phys. Rev. Lett. 79, 653-656.

Nakayama, Y., Kudo, K. \& Ohtsuki, T. [2010] "Variability and fluctuation in running gait cycle of trained runners and non-runners," Gait Posture 31, 331-335.

Nishikawa, J. \& Gohara, K. [2002] "Fractals in an electronic circuit driven by switching inputs," Int. J. Bifurcation and Chaos 12, 827-834.

Peng, C. K., Buldyrev, S. V., Havlin, S., Simons, M., Stanley, H. E. \& Goldberger, A. L. [1994] "Mosaic organization of DNA nucleotides," Phys. Rev. E 49, 1685-1689.

Peng, C. K., Havlin, S., Stanley, H. E. \& Goldberger, A. L. [1995] "Quantification of scaling exponents and crossover phenomena in nonstationary heartbeat time series," Chaos 5, 82-87.

Sato, S. \& Gohara, K. [2001] "Fractal transition in continuous recurrent neural networks," Int. J. Bifurcation and Chaos 11, 421-434.

Schmidt, R. A., Zelaznik, H., Hawkins, B., Frank, J. \& Quinn, J. T. J. [1979] "Motor output variability: A theory for the accuracy of rapid motor acts," Psychol. Rev. 86, 415-451.

Schmidt, R. A. [1991] Motor Learning and Performance: From Principles to Practice (Human Kinetics, Champaign). 
Schmiegel, A. \& Eckhardt, B. [1997] "Fractal stability border in plane couette flow," Phys. Rev. Lett. 79, $5250-5253$.

Suzuki, H. \& Yamamoto, Y. [2015] "Robustness to temporal constraint explains expertise in ball-over-net sports," Hum. Mov. Sci. 41, 193-206.

Tanii, K., Tachikawa, M., Tohei, T., Hong, F. L. \& Shimizu, T. [1991] "Instability and chaos in two-mode oscillation of a $\mathrm{CO}_{2}$ laser modulated by a saturable absorber," Phys. Rev. A 43, 1498-1501.

Tanii, K., Tohei, T., Sugawara, T., Tachikawa, M. \& Shimizu, T. [1999] "Two different routes to chaos in a two-mode $\mathrm{CO}_{2}$ laser with a saturable absorber," Phys. Rev. E 59, 1600-1604.

Torre, K., Deligniéres, D. \& Lemoine, L. [2007] " $1 / f^{\beta}$ fluctuations in bimanual coordination: An additional challenge for modeling," Exp. Brain Res. 183, 225234.

Wada, R. \& Gohara, K. [2001a] "Closures of fractal sets in non-linear dynamical systems with switched inputs," Int. J. Bifurcation and Chaos 11, 2205-2215.
Wada, R. \& Gohara, K. [2001b] "Fractal and closures of linear dynamical systems excited stochastically by temporal inputs," Int. J. Bifurcation and Chaos 11, 755-779.

Wallace, S. [1996] "Dynamic pattern perspective of rhythmic movement: An introduction," Advances in Motor Learning and Control, ed. Zelaznik, H. (Human Kinetics, Champaign, IL), pp. 155-194.

Werner, G. [2010] "Fractals in the nervous system: Conceptual implications for theoretical neuroscience," Front. Physiol. 6, 15.

Yamamoto, Y. \& Gohara, K. [2000] "Continuous hitting movements modeled from the perspective of dynamical systems with temporal input," Hum. Mov. Sci. 19, 341-371.

Zanone, P., Kostrubiec, V., Albaret, J. \& Temprado, J. [2010] "Covariation of attentional cost and stability provides further evidence for two routes to learning new coordination patterns," Acta Psychol. 133, 107-118. 